BMJ Open

Diabetes

Research

\& Care

\title{
Diabetes as an increasingly common comorbidity among patient hospitalizations for tuberculosis in the USA
}

\author{
Roula S Zahr, ${ }^{1}$ Ryan A Peterson, ${ }^{2}$ Linnea A Polgreen, ${ }^{3}$ Joseph E Cavanaugh, ${ }^{2}$ \\ Douglas B Hornick, ${ }^{1}$ Kevin L Winthrop, ${ }^{4}$ Philip M Polgreen ${ }^{5}$
}

To cite: Zahr RS, Peterson RA, Polgreen LA, et al. Diabetes as an increasingly common comorbidity among patient hospitalizations for tuberculosis in the USA. BMJ Open Diabetes Research and Care 2016;4:e000268. doi:10.1136/bmjdrc-2016000268

This work was presented as a poster at the American Diabetes Association 76th Scientific Sessions, 10-14 June 2016, New Orleans, Louisiana.

Received 17 May 2016 Revised 3 August 2016 Accepted 5 September 2016

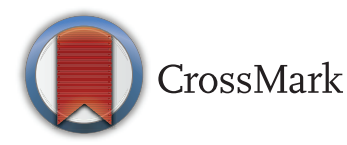

For numbered affiliations see end of article.

Correspondence to Dr Philip M Polgreen; philip-polgreen@uiowa.edu

\section{ABSTRACT}

Objective: Diabetes is a risk factor for active tuberculosis (TB). The purpose of this paper was to estimate the risk of hospitalization for TB with and without a secondary diagnosis of diabetes in groups with different ethnic backgrounds.

Research design and methods: We used the Nationwide Inpatient Sample from 1998 to 2011, identifying all patients with a primary diagnosis of TB and/or a secondary diagnosis of diabetes (type 1 or type 2) or HIV. Next, we performed logistic regression to investigate the association of diabetes status, HIV status, and race (and the interaction of diabetes and race) with the risk of hospitalization with a primary diagnosis of TB. We also included a time covariate, to determine whether potential risk factors changed during the study period.

Results: Controlling for HIV status, diabetes did not increase the odds of TB in white and black patients. However, in Hispanic and Asian/Pacific Islander patients, diabetes increased the odds of TB by a factor of $1.7(95 \% \mathrm{Cl} 1.51$ to 1.83$)$. Asian/Pacific Islanders who had diabetes but not HIV experienced 26.4 (95\% Cl 23.1 to 30.1$)$ times the odds of TB relative to the white males without diabetes or HIV. In addition, the percent of TB cases that belong to these high-risk groups (Asian/Pacific Islander/Hispanic diabetics) has more than doubled from $4.6 \%$ in 1998 to $9.6 \%$ in 2011.

Conclusions: In specific demographic groups, diabetes was a strong risk factor for hospital admissions for TB.

\section{INTRODUCTION}

The incidence of tuberculosis (TB) has decreased considerably in the USA during recent decades. ${ }^{1}{ }^{2}$ However, TB remains a major cause of morbidity and mortality worldwide. Globally, $>2$ billion people are infected with $\mathrm{TB},{ }^{3}$ and although the incidence of TB has slowly declined over the past decade, an estimated 9.6 million new cases occurred in 2014 alone. ${ }^{4}$ In the USA,

\section{Key messages}

- Diabetes is a risk factor for tuberculosis among hospitalized patients in the US.

- Admission rates for tuberculosis are not decreasing for patients with diabetes, and diabetes greatly increased the odds of a hospital admission for tuberculosis for Hispanic and Asian/ Pacific Islander patients.

- Bi-directional screening for diabetes and tuberculosis should be considered especially among patients immigrating from geographic areas where tuberculosis is common.

the majority of new cases of active TB now occur among people born outside the USA. ${ }^{2}$ Thus, TB disease trends in the USA will likely be affected by TB trends in other countries.

Worldwide, risk factors for active TB include exposure to tobacco smoke and indoor air pollution, ${ }^{5}$ overcrowding, ${ }^{6}$ and malnutrition. ${ }^{7}$ At an individual level, other comorbidities also increase the likelihood of developing active TB. One of the strongest known risk factors for developing active TB is HIV infection. High HIV prevalence rates are significantly correlated with high TB incidence rates, and most recent estimates suggested that $13 \%$ of the 9 million people who developed TB in 2013 were HIV-positive. ${ }^{8} 9$ In addition to HIV, diabetes has recently emerged as risk factor for developing active TB. ${ }^{10}$ Specifically, poor glucose control is associated with an increased risk of active TB. ${ }^{11}{ }^{12}$ Since the prevalence of diabetes is increasing in many countries where the burden of TB is high, controlling TB may become more difficult.

Estimating the contribution of diabetes to the incidence of $\mathrm{TB}$ over time is difficult because diabetes is not a reportable disease, and information about diabetes and glycemic control has not been routinely, universally, 
and systematically collected for patients with TB at the local level in the USA. Case report forms include diabetes as a comorbidity, but verification of the existence of diabetes may differ across geographic regions. Although the majority of TB care is performed in the outpatient setting, patients with more advanced cases of TB are hospitalized. The purpose of this paper was to estimate the frequency of admissions for a primary diagnosis of TB with and without a secondary diagnosis of diabetes, in order to better understand the association between TB and diabetes in the USA at a population level over time.

\section{METHODS}

\section{Data source}

We performed a cross-sectional study using the Nationwide Inpatient Sample (NIS). The NIS is maintained as part of the Healthcare Cost and Utilization Project by the Agency for Healthcare Research and Quality (AHRQ) ${ }^{13}$ and contains data from a $20 \%$ stratified sample of non-federal acute care hospitals. ${ }^{12}$ To adjust for yearly changes in the sampling design, we applied the discharge weights provided by AHRQ. ${ }^{13}$ Given the lack of personal identifiers in these data, the investigators' institutional review board determined that this project was not human subjects research. All analyses were performed using R, V.2.15.1 (R Foundation for Statistical Computing) and SAS, V.9.4.

We first identified all the hospitalizations over the period from January 1998 through December 2011 during which a primary diagnosis of TB was assigned, with the assumption that admission to a hospital with a primary diagnosis of TB represents active, rather than latent, TB. To ascertain cases, we used the International Classification of Diseases, 9th Revision, Clinical Modification (ICD-9-CM) codes 010-018. We then aggregated all cases by monthly and quarterly intervals to produce a national sample of cases of primary TB over time. All cases were assigned to a time interval on the basis of the month and year that the patient was admitted to the hospital. Next, we identified all hospitalizations over the same time period with a primary diagnosis of TB and a secondary diagnosis of diabetes. For diabetes and HIV case ascertainment, we used three digit codes 250 and 042, respectively. We included a national sample of all other hospitalizations in the study period in our analyses. For all included observations, we also extracted variables such as age, race, and gender as reported and defined in the NIS.

\section{Identifying incidence trends}

To gain a general sense of the patterns of incidence over time in each quarterly series (diabetes and diabetes-TB), we fit a regression model with a linear time trend and errors characterized as an autoregressive process. We used residual diagnostics to investigate the autocorrelation pattern in the residuals because failure to appropriately account for such autocorrelation may lead to incorrect inferential conclusions. The temporal correlation in the residuals was examined via the autocorrelation function and the partial autocorrelation function.

To determine how the demographics of primary hospitalizations for TB have changed over time, we compared race, age, and gender of cases between the years 1998 and 2011. Two-sample t-tests (for continuous variables) and Pearson's $\chi^{2}$ tests (for categorical variables) were used to assess whether each characteristic had changed from 1998 to 2011.

\section{Primary analysis}

Using monthly incidence data, we performed logistic regression to investigate how diabetic status, HIV status, and race (including race-diabetes and race-HIV interactions) are related to the risk of hospitalization with a primary diagnosis of TB. We also included a quantitative time covariate, along with time-diabetes, time-HIV, and time-race interactions, to account for whether these potential risk factors were changing during the study period. A simplified structural form for our model is as follows:

$$
\begin{gathered}
\text { TB status } \sim \text { Time }+ \text { DM }+ \text { Time } \times \text { DM }+ \text { HIV }+ \\
\text { Time } \times \text { HIV }+ \text { Race }+ \text { Time } \times \text { Race } \\
+ \text { DM } \times \text { Race }+ \text { HIV } \times \text { Race }
\end{gathered}
$$

Here, TB status refers to the binary outcome of whether or not an observation had a primary diagnosis of TB. Specifically, we are modeling the logit of the probability of a patient having a primary diagnosis of TB. Time is a quantitative variable coded in units of years, DM and HIV are binary variables that, respectively, indicate a secondary diagnosis of diabetes or HIV, and Race is a qualitative variable that designates one of five racial categories: white, black, Asian/Pacific Islander, Hispanic, and other. The fitted logistic regression model was used to produce model-based absolute incidence rate estimates for the most recent month in the study period (December 2011).

\section{RESULTS}

\section{Descriptive data}

After applying discharge weights, we observed 17844 discharges with a primary diagnosis of $\mathrm{TB}$, of which 3050 additionally had a secondary diagnosis of DM. We observed 74189360 discharges without a primary diagnosis of TB, of which 11750696 had a secondary diagnosis of DM. Prior to weighting, we excluded 7773 primary $\mathrm{TB}$ cases due to missing admission month (2775) or missing race (4998).

\section{Incidence trends}

Figure 1 shows the trends in quarterly incidence of primary admissions for $\mathrm{TB}$ and primary admissions for TB plus a secondary diagnosis of diabetes from January 1998 to December 2011. Admissions with a primary 
Figure 1 Quarterly

hospitalizations of patients diagnosed with primary TB (top)

or primary TB and secondary diabetes (bottom) in the USA (NIS, 1998-2011). *Not adjusted for autocorrelation; adjusted slope is -153.2 cases/year (based on modeling the errors as an autoregressive process of order one). NIS, Nationwide Inpatient Sample; TB, tuberculosis.
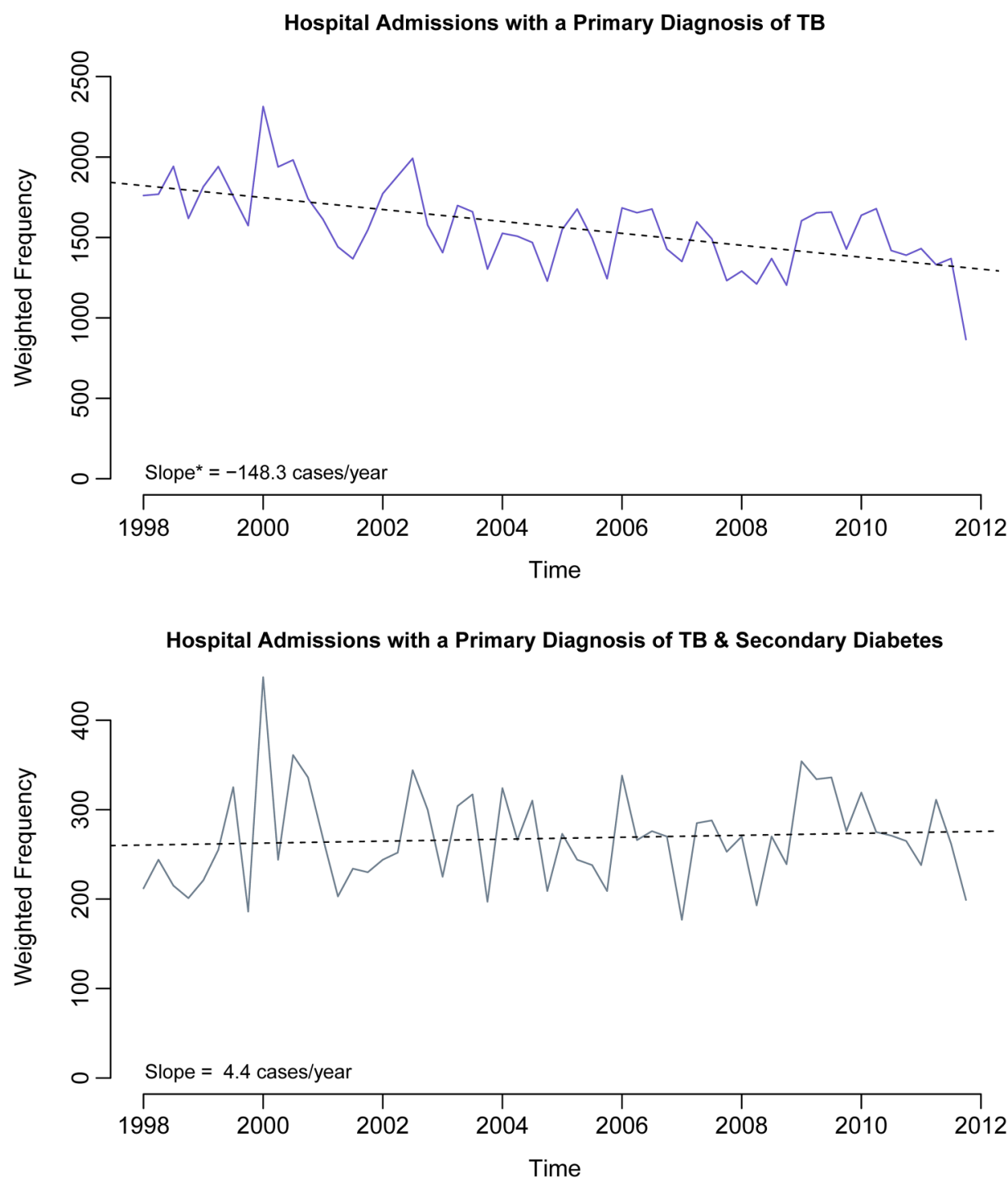

diagnosis of TB overall are decreasing by an average of about 148 cases per year (corrected for autocorrelation, this estimate is about 153 cases per year, $95 \%$ CI 85.3 to 221). However, we find no evidence of a substantive change in $\mathrm{TB}$ admissions over time for patients who have a secondary diagnosis of diabetes (an average of about 4.4 more cases per year, 95\% CI -9.2 to 18.0). This suggests that admissions with a primary diagnosis of $\mathrm{TB}$ are not significantly decreasing in the diabetic population.

Figure 2 shows the extent to which all inpatient admissions with secondary diabetes are increasing over time in each race category. In figure 3, for selected risk profiles, including Asian/Pacific Islanders and Hispanic populations with a secondary diagnosis of diabetes, we show how the proportion of admissions with a primary diagnosis of TB has changed since 1998. While the proportion of TB associated with patients with HIV is decreasing considerably, it is increasing for Asian/Pacific Islander and Hispanic patients. All trends in figures 2 and 3 are statistically significant at the 0.001 level. In addition, we found no significant changes in mean patient age $(\mathrm{p}=0.19)$ or patient gender $(\mathrm{p}=0.48)$ over this time period.

\section{Main results}

We present the results from our logistic regression model in table 1. Our model is estimating the odds of a primary diagnosis of $\mathrm{TB}$ given a patient's set of risk factors (race, HIV, DM, and time of admission). We designate white patients without a secondary diagnosis of diabetes or HIV as the baseline category. The table presents estimated ORs for all combinations of race and diabetic status relative to this baseline category. All results are based on the most recent month in the study period (December 2011). We found that race is a major component of TB risk: in the absence of a secondary diabetes diagnosis, all admissions among non-white patients have approximately fivefold the odds of a primary diagnosis of $\mathrm{TB}$, and Asian/Pacific Islanders have 16.2 (95\% CI 14.6 to 17.9 ) times the odds of a primary diagnosis of TB. Paradoxically, based on the 
Figure 2 Secondary diabetes admissions for white, black, Hispanic, and Asian/Pacific Islander populations in the National Inpatient Sample.
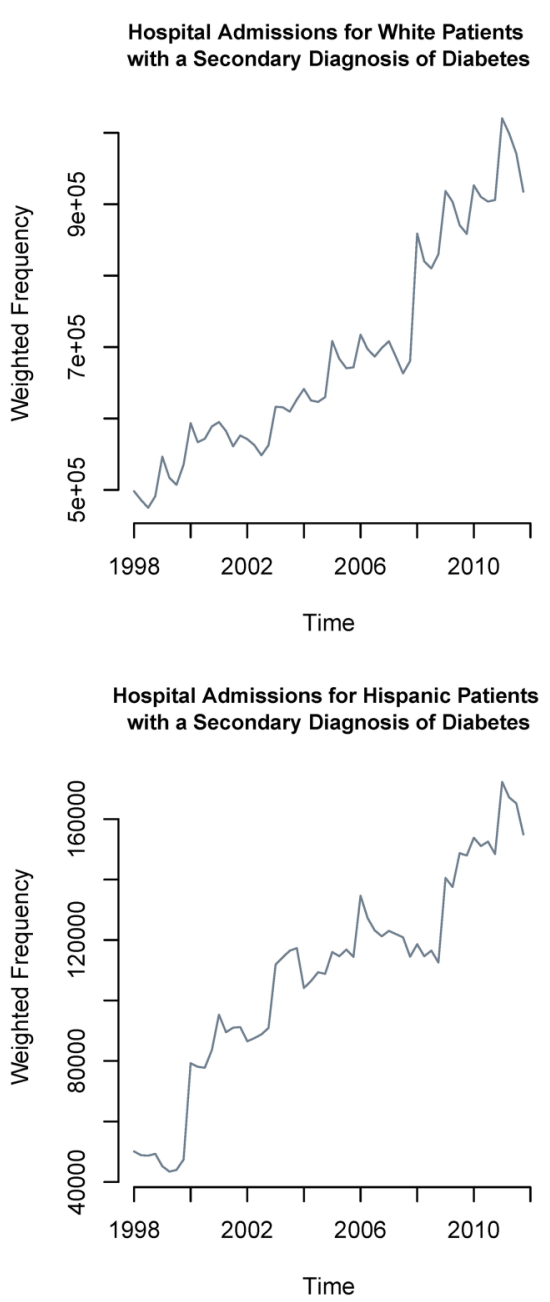

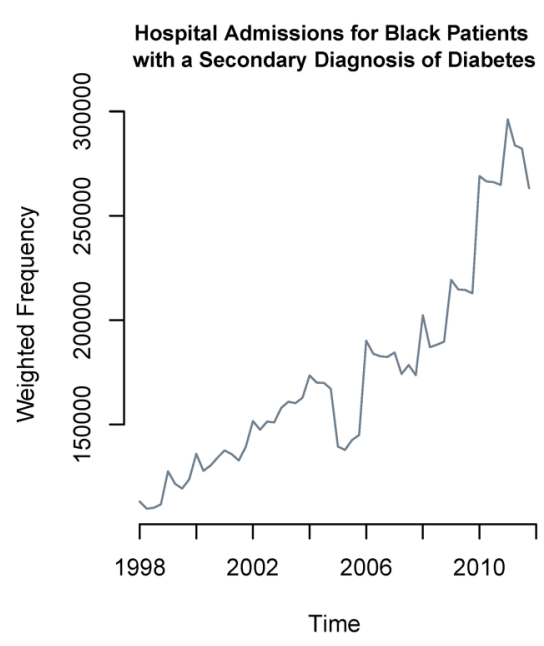

Hospital Admissions for Asian/PI Patients with a Secondary Diagnosis of Diabetes

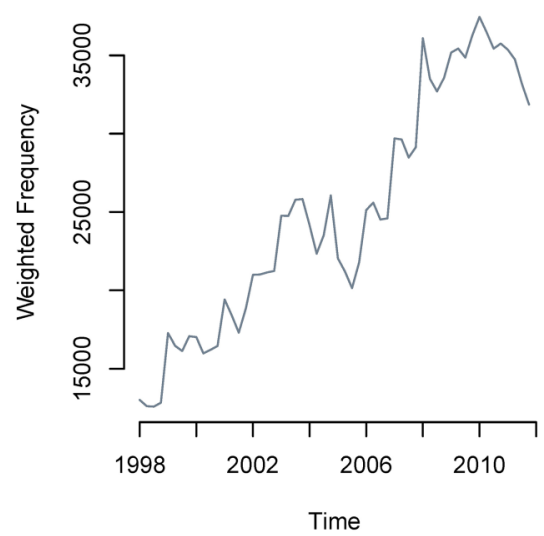

ORs for primary TB in white and black patients, diabetes appears protective. For white patients, those with a secondary diagnosis of DM had $28.8 \%$ (95\% CI $20.1 \%$ to $36.6 \%$ ) reduced odds of having a primary diagnosis of TB. Black patients with a secondary DM diagnosis had $235.5 \%$ (95\% CI $27.8 \%$ to $42.4 \%$ ) reduced odds of having a primary diagnosis of TB. However, within Hispanic and Asian/Pacific Islander subgroups, diabetes increases the odds of a primary diagnosis of TB by $65.9 \%$ (95\% CI $50.7 \%$ to $82.7 \%$ ) and $63.6 \%$ (95\% CI $46.4 \%$ to $82.9 \%$ ), respectively. Finally, relative to the healthy white male baseline, Asian/Pacific Islanders who have diabetes experience 26.4 (95\% CI 23.1to 30.1) times the odds of TB.

In figure 4, we present the estimated absolute risk of a primary diagnosis of TB for each of our study populations (per 10000 total hospitalizations). The estimates pertain to the most recent month in the study period, December 2011. From the figure, it is clear that there are certain populations at increased risk for TB. Of note, Asian/Pacific Islanders with secondary diabetes have a significantly higher absolute risk of TB than white patients with HIV $(\mathrm{p}<0.001)$.

\section{DISCUSSION}

Our results demonstrate that hospitalizations for patients with a primary diagnosis of TB have decreased dramatically in the USA. However, to the contrary, the incidence for patients with a primary diagnosis of $\mathrm{TB}$ and a secondary diagnosis of diabetes has not decreased. Furthermore, while HIV has been recognized as the strongest known risk factor for developing active $\mathrm{TB}$, we show that during our study period the frequency of HIV as a secondary diagnosis among patients admitted with a primary diagnosis of $\mathrm{TB}$ has decreased from $5.35 \%$ to $1.92 \%$. The frequency of diabetes as a secondary diagnosis has increased from $11.2 \%$ to $19.5 \%$ of all hospitalized cases. Thus, our results provide evidence that diabetes is emerging as an important comorbidity in patients hospitalized with TB. In fact, among Asian/Pacific Islanders, diabetes appears to be a stronger risk factor than HIV.

The association between diabetes and TB has been known for centuries. Ancient works describe the symptoms of patients who progressed from obesity to thirst, to glycosuria, and ultimately to unconsciousness or TB. ${ }^{14}$ More recently, the close association between diabetes and $\mathrm{TB}$ has been demonstrated in multiple 


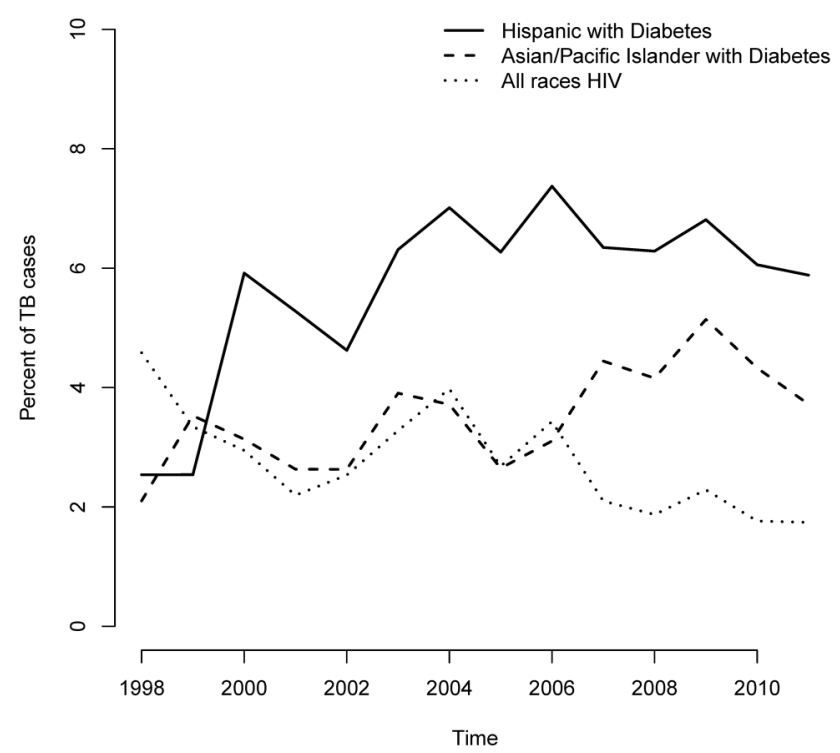

Figure 3 Yearly trends in percentage of admissions for primary TB cases that occurred in selected high-risk groups from 1992 to 2011: secondary diagnoses of HIV are falling, while secondary diagnoses of diabetes are rising. Cases were aggregated by year and broken down into categories based on HIV status, DM status, and race. All trends shown were tested based on a linear probability model and found to be statistically significant at the 0.001 level. DM, diabetes mellitus; TB, tuberculosis.

Table 1 Estimated* ORs and $95 \%$ Cls for a primary diagnosis of TB by race category (white, black, Hispanic, Asian/Pacific Islander, and other), and diabetic status for patients without a secondary diagnosis of HIV (white, non-diabetic patients are the comparison group)

\begin{tabular}{lcc}
\hline Group & $\begin{array}{l}\text { No secondary } \\
\text { diagnosis of } \\
\text { diabetes }\end{array}$ & $\begin{array}{l}\text { Secondary } \\
\text { diagnosis of } \\
\text { diabetes }\end{array}$ \\
\hline White & 1 (baseline) & $0.7(0.6$ to 0.8$)$ \\
Black & $4.3(3.9$ to 4.7$)$ & $2.7(2.4$ to 3.1$)$ \\
Hispanic & $5.7(5.3$ to 6.3$)$ & $9.6(8.5$ to 10.8$)$ \\
Asian/Pacific & $16.2(14.6$ to 17.9$)$ & $26.4(23.1$ to 30.1$)$ \\
Islander & $5.7(5.1$ to 6.5$)$ & $6.7(5.6$ to 7.9$)$ \\
Other &
\end{tabular}

${ }^{*}$ ORs were estimated using logistic regression for the year ended 2011, and assume patients do not have HIV.

TB, tuberculosis.

reports, and it is reconfirmed in this study. In a systematic review of $\mathrm{TB}$ studies, diabetes appears to increase the risk of developing TB disease about threefold. ${ }^{15}$ In addition to the increased risk of TB occurrence, patients with TB and diabetes have higher baseline bacterial burdens, longer time to conversion after treatment, and higher risk for multidrug resistance, treatment failure, recurrence, and death compared to patients with TB without diabetes. $^{16-20}$

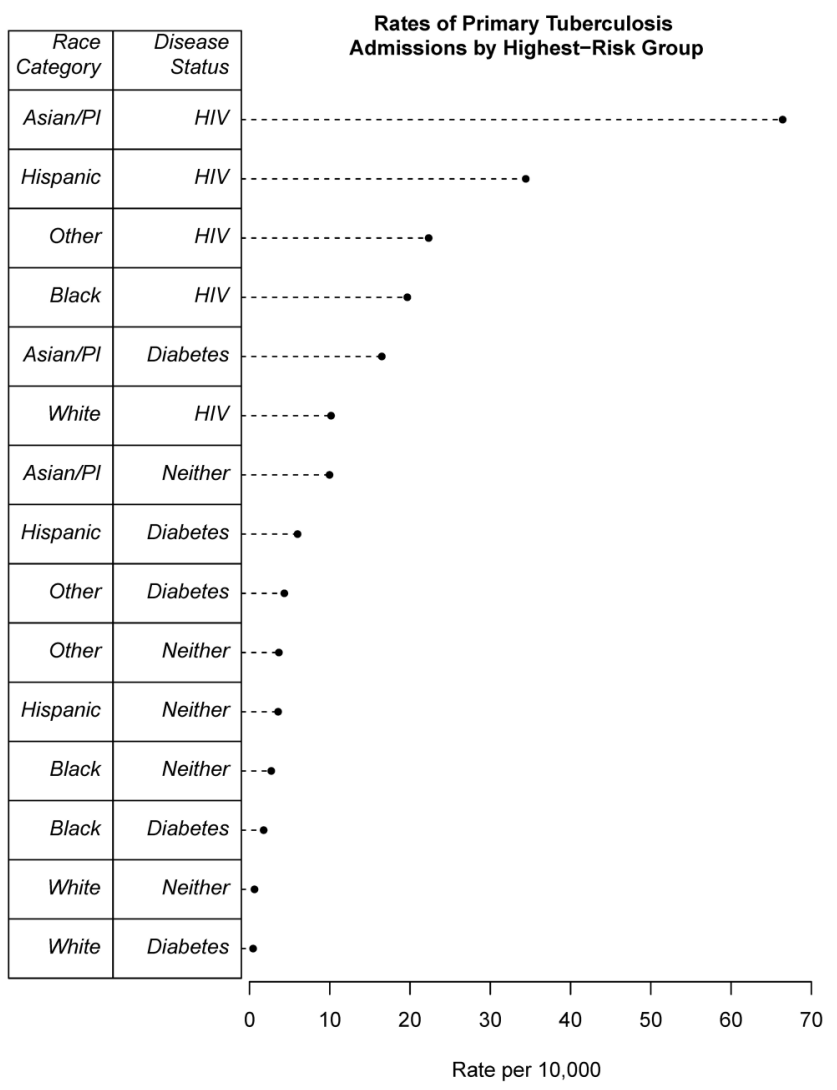

Figure 4 Estimated absolute incidence rates of primary TB admissions (per 10000 total hospitalizations) by race category (Asian/Pacific Islander, Hispanic, black, white, other) and secondary disease status (diabetic, HIV, neither). Rates calculated from transforming model-based odds estimates into probability estimates. TB, tuberculosis.

In general, the risk for developing active disease is related to diabetic control rather than the disease state itself. For example, in the Philadelphia Diabetic Survey, Boucot et al found a twofold increase in prevalent TB in patients with diabetes compared to controls with similar demographic characteristics. In addition, patients who needed $>40$ units of insulin per day were twice as likely to develop TB as those using lower doses. ${ }^{21}$ Also, patients with $\mathrm{HbA} 1 \mathrm{C}>7 \%$ had an increased risk for $\mathrm{TB}$ than patients with $\mathrm{HvA} 1 \mathrm{C}<7 \%{ }^{11}{ }^{12}$ Finally, the probability of developing TB was substantially higher in insulindependent diabetics than non-insulin-dependent diabetics. $^{22} 23$ The exact mechanism by which diabetics are at increased risk for TB is not known, but changes in innate and adaptive immunity are likely to play a role. ${ }^{24-30}$

Given the close relationship between TB and diabetes, the WHO and the International Union Against Tuberculosis and Lung Disease launched the Collaborative Framework for Care and Control of Tuberculosis and Diabetes Mellitus in 2011. This initiative stresses the importance of bidirectional screening for these two important diseases: screening for TB in patients with diabetes and screening for diabetes in patients diagnosed with TB. The goal of bidirectional 
screening is to improve early diagnosis rates and treatment outcomes for both diseases. In general, systematic screening for TB in persons with diabetes has only been recommended in countries with relatively high TB rates because the number needed to screen in order to detect a new case of TB is high when TB prevalence is low. However, our results suggest that even in the USA, a country with a relatively low and decreasing incidence of $\mathrm{TB}$, bidirectional screening may be warranted in specific high-risk populations in hospital settings.

In the USA, the greatest numbers of immigrants originate from Mexico, China, India, and the Philippines. ${ }^{31}$ All of these countries have TB prevalence rates that are much higher than in the USA. ${ }^{4}$ Worldwide, India and China alone accounted for $24 \%$ and $11 \%$, respectively, of the estimated 9 million people who developed TB in 2013. ${ }^{4}$ In addition, diabetes rates have increased dramatically in all of these countries. Now, India and China have diabetes rates comparable to the USA, and diabetes is even more common in Mexico. In addition, many immigrants may be at additional risk for developing diabetes after moving to the USA given the risk factors for obesity and diabetes common in many parts of the USA (eg, urbanization, nutrition transition to unhealthy diet, and a more sedentary lifestyle).

Although we cannot measure the effects of immigration in our study, we did observe a substantial shift in demographic patterns among our patient population. Specifically, a recorded race of Hispanic or Asian was much more common in 2011 than in 1998 among patients admitted with a primary diagnosis of $\mathrm{TB}$. In contrast, patients with a recorded race of white or black were much less common. Thus, immigration trends and travel patterns will become increasingly important considerations in the efforts to control the epidemics of both diseases mainly through bidirectional screening and integrated management.

Screening and preventive medicine, especially for diabetes care, is often performed in ambulatory settings. Our hospital-based results show that physicians who primarily practice in inpatient settings should also consider bidirectional screening for TB and diabetes. It is essential to detect patients with active TB or at risk for active TB promptly: many hospital outbreaks are associated with the delayed recognition of $\mathrm{TB},{ }^{32} 33$ and in the USA, many opportunities to diagnose TB are missed. ${ }^{34-36}$ Our results suggest that clinicians should consider TB among diabetic patients with TB-like symptoms (chronic cough, weight loss, etc), especially among diabetic patients from high-risk groups. Finally, our model did show that patients who were white with a secondary diagnosis of diabetes were less likely to be admitted with a primary diagnosis of TB. However, we think that this result is a function of decreased risk for acquiring TB in terms of exposure to the disease. Alternatively, white patients with diabetes may have access to better healthcare and thus have better diabetes control. It is also possible that patients with diabetes are more likely to receive close medical care, and if they have $\mathrm{TB}$, they might be less likely to progress to severe active $\mathrm{TB}$ cases that require hospitalization.

Our study has several limitations. First, we exclusively use administrative data; thus, we cannot review patients' medical records to confirm the accuracy of the diagnostic codes assigned to the patient. For example, we cannot validate the diagnosis by determining whether patients diagnosed with TB were given TB-related therapy. However, any misclassification of TB cases should not differ between diabetics and non-diabetics. We acknowledge the limitation of using ICD-9 codes to define diabetes as a secondary diagnosis. Specifically, there is a possibility of unreported disease and other codes, reflecting the diagnoses of hyperglycemia or insulin use, may be used. However, this limitation applies to all groups over time. Since we are using administrative data, we also do not know the level of glycemic control for those assigned a diagnosis of diabetes. However, our use of administrative data allowed us to examine disease trends over a long period of time across the entire country. Second, we only focus on patients hospitalized with TB. The vast majority of TB cases are not hospitalized. Nevertheless, our study population is important. By focusing on hospitalized patients, we focus on patients with more advanced disease. Also, hospitalized patients are an important population to consider for enhanced screening because if these patients are not diagnosed and placed in isolation, then they are likely to transmit contagious infectious diseases to healthcare workers and other patients, given the contact patterns within hospitals. ${ }^{37} 38$ Third, the sensitivity and specificity of TB codes is less than perfect. However, we restrict our analysis to patients with a primary diagnosis of TB and thus are much less likely to include patients who received this diagnostic code improperly, for example, for receiving a TB diagnostic test only. Finally, our data source does not allow us to determine where someone was exposed to $\mathrm{TB}$ or whether they had traveled or were born outside the USA. We can only consider the race as recorded in the administrative database.

Despite these limitations, we demonstrate that the worldwide diabetes epidemic will likely influence future TB-control efforts. Furthermore, we suggest that targeted bidirectional screening may be especially important for hospitalized patients. Future investigations should attempt to investigate the yield of such targeted screening programs. Finally, our results highlight the importance of considering TB in patients with diabetes admitted to the hospital and for screening for diabetes in patients diagnosed with TB, especially if patients originate from regions where $\mathrm{TB}$ is more common.

\section{Author affiliations}

${ }^{1}$ Department of Internal Medicine, University of lowa, lowa City, Iowa, USA

${ }^{2}$ Department of Biostatistics, University of lowa, lowa City, lowa, USA

${ }^{3}$ Department of Pharmacy Practice and Science, University of lowa, lowa City, lowa, USA 
${ }^{4}$ Department of Infectious Diseases, Ophthalmology, Public Health and Preventive Medicine, Oregon Health and Science University, Portland, Oregon, USA

${ }^{5}$ Departments of Internal Medicine and Epidemiology, University of lowa, lowa City, Iowa, USA

Contributors RSZ analyzed data and wrote the manuscript. RAP analyzed data and contributed to methods and results. LAP. analyzed data, contributed to discussion and reviewed/edited the manuscript. JEC contributed to methods and results. DBH contributed to discussion and reviewed/edited the manuscript. KLW contributed to discussion and reviewed/edited the manuscript. PMP (guarantor) analyzed data and wrote the manuscript.

Funding This work was supported by the National Heart, Lung, and Blood Institute, grant number K25HL122305; National Institutes of Health, Training in Kidney Disease, Hypertension, Cell Biology, grant number T32DK007690; and University of lowa Health Venture's Signal Center.

Competing interests None declared.

Provenance and peer review Not commissioned; externally peer reviewed.

Data sharing statement Nationwide Inpatient Sample (NIS) data were used for this study. The NIS is maintained as part of the Healthcare Cost and Utilization Project (HCUP) by the Agency for Healthcare Research and Quality (AHRQ). NIS data can be obtained from AHRQ.

Open Access This is an Open Access article distributed in accordance with the Creative Commons Attribution Non Commercial (CC BY-NC 4.0) license, which permits others to distribute, remix, adapt, build upon this work noncommercially, and license their derivative works on different terms, provided the original work is properly cited and the use is non-commercial. See: http:// creativecommons.org/licenses/by-nc/4.0/

\section{REFERENCES}

1. Yates T, Haffner SM, Schulte PJ, et al. Association between change in daily ambulatory activity and cardiovascular events in people with impaired glucose tolerance (NAVIGATOR trial): a cohort analysis. Lancet 2014;383:1059-66.

2. Centers for Disease Control and Prevention. Tuberculosis (TB) data and statistics. http://www.cdc.gov/tb/statistics/default.htm (cited 4 Oct 2015).

3. Lonnroth K, Raviglione M. Global epidemiology of tuberculosis: prospects for control. Semin Respir Crit Care Med 2008;29:481-91.

4. World Health Organization. Global tuberculosis report, 2015. http:// www.who.int/tb/publications/global report/en/ (cited 6 Jul 2016).

5. Lin HH, Ezzati M, Murray M. Tobacco smoke, indoor air pollution and tuberculosis: a systematic review and meta-analysis. PLoS Med 2007; 4:e20.

6. Baker M, Das D, Venugopal K, et al. Tuberculosis associated with household crowding in a developed country. J Epidemiol Community Health 2008;62:715-21.

7. Cegielski JP, McMurray DN. The relationship between malnutrition and tuberculosis: evidence from studies in humans and experimental animals. Int J Tubercul Lung Dis 2004;8:286-98.

8. Geldmacher C, Zumla A, Hoelscher M. Interaction between HIV and Mycobacterium tuberculosis: HIV-1-induced CD4 T-cell depletion and the development of active tuberculosis. Curr Opin HIV AIDS 2012;7:268-75.

9. Lai RP, Nakiwala JK, Meintjes G, et al. The immunopathogenesis of the HIV tuberculosis immune reconstitution inflammatory syndrome. Eur J Immunol 2013;43:1995-2002.

10. Dooley KE, Chaisson RE. Tuberculosis and diabetes mellitus: convergence of two epidemics. Lancet Infect Dis 2009;9:737-46.

11. Leung CC, Lam TH, Chan WM, et al. Diabetic control and risk of tuberculosis: a cohort study. Am J Epidemiol 2008;167:1486-94.

12. Chiang $\mathrm{CY}$, Bai $\mathrm{KJ}$, Lin $\mathrm{HH}$, et al. The influence of diabetes, glycemic control, and diabetes-related comorbidities on pulmonary tuberculosis. PLOS ONE 2015;10:e0121698.

13. Agency for Healthcare Research and Quality. Nationwide inpatient sample trends supplemental (nis-trends) files, 16 Aug 2012. http:// www.hcup-us.ahrq.gov/nisoverview.jsp
14. Rajalakshmi S, Veluchamy G. Yugi's pramegam and diebetes mellitus: an analogue. Bull Indian Inst Hist Med Hyderabad 1999;29:83-7.

15. Jeon CY, Murray MB. Diabetes mellitus increases the risk of active tuberculosis: a systematic review of 13 observational studies. PLoS Med 2008;5:e152.

16. Baker MA, Harries $A D$, Jeon $\mathrm{CY}$, et al. The impact of diabetes on tuberculosis treatment outcomes: a systematic review. BMC Med 2011;9:81.

17. Gomez-Gomez A, Magaña-Aquino M, López-Meza S, et al. Diabetes and other risk factors for multi-drug resistant tuberculosis in a Mexican population with pulmonary tuberculosis: case control study. Arch Med Res 2015;46:142-8.

18. Guler M, Unsal E, Dursun B, et al. Factors influencing sputum smear and culture conversion time among patients with new case pulmonary tuberculosis. Int J Clin Pract 2007;61:231-5.

19. Jimenez-Corona ME, Cruz-Hervert LP, García-García L, et al. Association of diabetes and tuberculosis: impact on treatment and post-treatment outcomes. Thorax 2013;68:214-20.

20. Wang CS, Yang CJ, Chen HC, et al. Impact of type 2 diabetes on manifestations and treatment outcome of pulmonary tuberculosis. Epidemiol Infect 2009;137:203-10.

21. Boucot KR, Dillon ES, Cooper DA, et al. Tuberculosis among diabetics: the Philadelphia survey. Am Rev Tuberc 1952;65:1-50.

22. Olmos P, Donoso J, Rojas N, et al. [Tuberculosis and diabetes mellitus: a longitudinal-retrospective study in a teaching hospital] Rev Med Chil 1989;117:979-83.

23. Swai AB, McLarty DG, Mugusi F. Tuberculosis in diabetic patients in Tanzania. Trop Doct 1990;20:147-50.

24. Gomez DI, Twahirwa M, Schlesinger LS, et al. Reduced Mycobacterium tuberculosis association with monocytes from diabetes patients that have poor glucose control. Tuberculosis (Edinb) 2013;93:192-7.

25. Lecube A, Pachón G, Petriz J, et al. Phagocytic activity is impaired in type 2 diabetes mellitus and increases after metabolic improvement. PLOS ONE 2011;6:e23366.

26. Liu HF, Zhang HJ, Hu QX, et al. Altered polarization, morphology, and impaired innate immunity germane to resident peritoneal macrophages in mice with long-term type 2 diabetes. J Biomed Biotechnol 2012;2012:867023.

27. Sun $\mathrm{C}$, Sun $\mathrm{L}, \mathrm{Ma} \mathrm{H}$, et al. The phenotype and functional alterations of macrophages in mice with hyperglycemia for long term. J Cell Physiol 2012;227:1670-9.

28. Hodgson K, Morris J, Bridson T, et al. Immunological mechanisms contributing to the double burden of diabetes and intracellular bacterial infections. Immunology 2015;144:171-85.

29. Martinez N, Kornfeld $\mathrm{H}$. Diabetes and immunity to tuberculosis. Eur J Immunol 2014;44:617-26.

30. Restrepo BI, Schlesinger LS. Impact of diabetes on the natural history of tuberculosis. Diabetes Res Clin Pract 2014;106:191-9.

31. Walters NP, Trevelyan EN. The newly arrived foreign-born population of the United States. 2010 American Community Survey Briefs, 2011. http://www.census.gov/content/dam/Census/library/ publications/2011/acs/acsbr10-16.pdf (cited 27 Sep 2015).

32. Kenyon TA, Ridzon R, Luskin-Hawk R, et al. A nosocomial outbreak of multidrug-resistant tuberculosis. Ann Intern Med 1997;127:32-6.

33. Zaza S, Blumberg HM, Beck-Sagué C, et al. Nosocomial transmission of Mycobacterium tuberculosis: role of health care workers in outbreak propagation. J Infect Dis 1995;172:1542-9.

34. Miller AC, Polgreen LA, Cavanaugh JE, et al. Missed opportunities to diagnose tuberculosis are common among hospitalized patients and patients seen in emergency departments. Open Forum Infect Dis 2015;2:ofv171.

35. Guderian LJ, Miller WC, Seña AC, et al. Increased prevalence of advanced tuberculosis in rural low tuberculosis caseload counties in North Carolina. Int J Tuberc Lung Dis 2011;15:1455-60, i.

36. Wallace RM, Kammerer JS, lademarco MF, et al. Increasing proportions of advanced pulmonary tuberculosis reported in the United States: are delays in diagnosis on the rise? Am J Respir Crit Care Med 2009;180:1016-22.

37. Curtis DE, Hlady CS, Kanade G, et al. Healthcare worker contact networks and the prevention of hospital-acquired infections. PLoS ONE 2013;8:e79906.

38. Hornbeck T, Naylor D, Segre AM, et al. Using sensor networks to study the effect of peripatetic healthcare workers on the spread of hospital-associated infections. J Infect Dis 2012;206:1549-57. 